5. Duranta coriacea Hayek, nov. spec.

Rami subtetragoni glabri aculeati. Folia opposita breviter petiolata in petiolum attenuata elliptica acuta integerrima, margine parum revoluta, coriacea, glabra, concoloria, plana, supra nitida subtus opaca. Racemi erecti floribus nutantibus. Calyx tubulosus breviter 5-dentatus margine parum pubescenti-tomentosus. Corolla tubo fere recto limbo plano, utrinque tenuiter pubescenti trmentella. Drupa carnosa diam. $8 \mathrm{~mm}$ lata.

Differt a Duranta Plumieri foliis coriaceis nitidis semper integerrimis, a D. Mutisii foliis planis laevibus, nec nerroso-rugosis, concoloribus.

Nouvelle Grenade, Prov. de Cauca: Valleo de Cauca, $1000 \mathrm{~m}$ (Triana 2074). -- Cauca (Karsten). - Andes Quitensis: Pallatanga (Spruce 5574).

6. Duranta tomentosa Hayek, nor. spec.

Rami subtetragoni inermes cum pedunculis dense crispo-tomentosi. Folia breviter pedunculata, ovata, acuta, integerrima, vel apicem versus remote serrulata, coriacea, supra nitida, grosse nervoso-rugosa et pilis crispulis obsita, demum glabrescentia, subtus dense crispule tomentosa (in siceo ferruginea). Racemi axillares simplices vel ramosi, floribus nutantibus. Calyx breviter 5 -dentatus, breviter crispo-villosus. Corolla tubo elongato curvat" $10-12 \mathrm{~mm}$ longo, limbo patulo magnitudine fere iis Durantae Plumieri.

Similis Durantae Mutisii, sed indumento valde diversa.

Nouvelle-Grenade: Prov. de Pasto, $2600 \mathrm{~m}$ (Triana 2073).

7. Callicarpa elegans Hayek, nov. spec.

Rami subtetragoni, iuvenes tomento floccoso obtecti, adulti glabri. Folia coriacea brevissime petiolata, in petiolum attenuata, lanceolata, in apicem linearem lingam acutam producta, margine regulariter serrata, glaberrima, in pagina superiore sparse, in pagina inferiore densius punctis glandulosis aureis nitidjs obsita. Crmae pauciflorae graciles pedunculis petiolum duplo fere superantibus, dimidiam vel tertiam partem foliorum aequantes, pedunculis pedicellisque tomento sparso deciduo obtectis.

Foliorum forma Callicarpae caudatae Maxim. simillima, differt foliorum glabritie et serrutura maiore nec non indumento ramorum diverso; a $C$. purpurea Juss. foliis coriaceis nec chartaceis in acumen elongatum productis, nitidis, dentatis nec serratis, punctis glandulosis aureis nitidis nec purpureis opacis diversa est.

Ins. Philippines (Cumming 1460).

\title{
XXII. Some new South American Species of Koeleria.
}

By Dr. K. Domin (Prague).

(Originaldiagnosen.)

The descriptions of some new Koeleria Species from South America are contained in the following article. 'These are for the most part native 
plants of Argentine and can be found in the Herbarium of the Royal Botanical Museum in Berlin and partly in the Herbarium of the Royal Botanic Gardens in kew near London.

$I$ restrict myself in the following article to the descriptions and short remarks; details of their systematical position can by found in my general monography of this genus, which will shortly appear.

1. Koeleria Hieronymi Domin, nov. spec.

Perennis laxe caespitosa culmis interdum solitariis, vaginis vetustis intus argenteo-nitidulis infimis retrorsum patentibus, culmis valde elatis usque $9 \mathrm{dm}$ altis robustis sed valde mollibus parte tertia superiori nudis et totis dense molliter cano-pubescentibus, foliis radicalibus paucis sub flore saepe subsiccis vaginis patentibus instructis, culmis circa $3-4$-foliatis, foliis, culmeis vaginis longis pallidis tenuibus culmos laxe amplectentibus vel totis vel parte superiori subpatentibus striatis molliter sublaxe pubescentibus, laminis longioribus (circa $1-1,5 \mathrm{dm}$ ) planis tenuibus valde latis ( $3 \mathrm{~mm}$ usque plus $4 \mathrm{~mm}$ ) subglaucescentibus sat mollibus saepius fere glabris parum scabris ad marginem scabriusculum breviter ciliatulis instructis, ligulis glabris laceroso-incisis protractis $1,5-3 \mathrm{~mm}$ longis, paniculis cylindricis c. $10-13 \mathrm{~cm}$ longis et tantum c. $8-10 \mathrm{~mm}$ latis inferne semper interruptis albescenti-nitidulis densissimis haud lobatis vel cum parum lobatis tunc lobis brevibus densissimis, spiculis fere sessilibus vel brevissime petiolulatis parvis pallidis lineari-lanceolatis c. $4 \mathrm{~mm}$ longis bifloris, glumis longitudine latitudineque inaequalibus glaberrimis late scarioso-marginatis, superiori c. $4 \mathrm{~mm}$ longa acutata vel in apicem breviter mucroniformem protracta flosculis parum breviori, inferiori angustiori acuminata tantum c. $3 \mathrm{~mm}$ longa flosculis conspicue breviori, glumellis lineari-lanceolatis glabris superne scarioso-marginatis acutatis interdum breviter mucronatulis vel paulo sub apice aristulis dorsalibus minimis apicem glumellarum vix attingentibus et numquam superantibus munitis, flosculo secundo mediocriter longe pedunculato, rhachide breviter protracta glabra vel subglabra, paleis glumellis paulo brevioribus apice breviter bidentatulis.

Floret Januario.

Exsicc.: G. Hieronymus et G. Niederlein: Flora Argentina no. 703.

Habitat in Argentina: En las cercanias del Pié de la buesta, mas arriba del Vallecito; Sierra Famatina, Prov. de la Rioja. Leg. 15.-20. I. 1879 G. Hieronymus et G. Niederlein (H. Berol.).

Species egregia et cum florae Argentinae Koeleriis sequentibus formis intermediis haud conjuncta esse videtur. Caespitibus laxis, colore canescenti, statura robusta, culmis mollibus pubescentibus, vaginis subpatentibus molliter pubescentibus, ligulis protractis, panicula densissima angusta, spiculis parvis facile dignoscitur.

2. Koeleria argentina Domin, nov. spec.

Pluriculmis sed minus dense caespitosa vaginis infimis sub flore subaphyllis tenuibus saepe subpatentibus nitidulis, culmis gracilibus c. $4-5 \mathrm{dm}$. 
altis totis retrorsum tenuiter pubescentibus, foliis radicalibus culmeisque infimis elongatis usque $2 \mathrm{dm}$ longis et culmos dimidios aequantibus plurimis planis $1-2 \mathrm{~mm}$ latis longe tenuiter acuminatis glabris scabriusculis glaucis haud rigidis. raginis glabris, foliis culmeis pluribus planis unacum vaginis glabris culmum arete amplectentibus parte suprema pro more apertis, ligulis omnibus elongatis c. $2 \mathrm{~mm}$ longis parum incisis glabris; paniculis oblongo-cylindricis nitentibus c. $6-8 \mathrm{~cm}$ longis valde laxis lobatis sed ramis depauperatis remotis, spiculis breviter sed conspicue pedunculatis c. $5,25-6 \mathrm{~mm}$ longis pallidis trifloris, glumis longitudine inaequalibus flosculis valde brevioribus superiori c. $3.5 \mathrm{~mm}$ longa latiori in apicem longe acuminatam protracta inferiori lineari acuminata c. $2,5 \mathrm{~mm}$ longa flosculis saepe dimidio breviori utraque glaberrima magna ex parte scariosa, glumella flosculi primi secundique lineari-lanceolata subacuminata haud raro apice breviter mucronatula virescenti-pallida superne margine angustiori hyalino cincta, paleis glumellis tertia parte brevioribus apice conspicue bidentatis. flosculo tertio diminuto (sed semper regulariter evoluto) longe pedunculato. pedunculo flosculo aequilongo, rhachide protracta glabra.

Floret Januario.

Habitat in Argentina: En las cercanias del Pit de la buesta, mas arriba del Vallecito: Sierra Famatina, Prov. de la Rioja. Leg. d. 15.--20. I. 1879 G. Hieronymus et G. Niederlein (H. Berol.).

Species meo ex sensu optima! Excellit glabritie, culmis gracilibus totis retrorsum molliter puberulis, paniculis valde laxis, imprimis autem spicularum formatione.

3. Koeleria Bergii Hieron. in Bol. de la Acad. Nac. Curdoba 1880 , III. entr. IV., p. 50; Domin ampl.

Perennis, culmis robustis exaltatis usque plus $5 \mathrm{dm}$ altis totis glabris usque infra apicem foliatis, foliis culmeis laminis planis sat latis (juxta vaginam circiter $3-4 \mathrm{~mm}$ ) longiusculis (usque $15 \mathrm{~cm}$ longis) pubescentibus instructis, vaginis omnibus pilis reversis vel subreversis molliter pubescentibus saepius elongatis, ligulis brevibus usque $1,5 \mathrm{~mm}$ longis, lamina folii culmei supremi paniculam saepe attingente, paniculis subdensis c. $8-10 \mathrm{~cm}$ longis inferne lobatis nitentibus, spiculis breviter sed conspicue pedunculatis linearibus c. $5,5--6 \mathrm{~mm}$ longis tri- vel bifloris, glumis (praecipue gluma superiori) flosculos longitudine adaequantibus vel iis tantum paulo brevioribus acuminatis subaequilongis, inferiori duplo angustiori lineari acuminata c. $4,75-5 \mathrm{~mm}$ longa, superiori acuminata lineari-lanceolata c. $6 \mathrm{~mm}$ longa, utraque glaberrima exacte (carina quoque) laevi et maxima ex parte scariosa. flosculis breviter pedunculatis, glumellis linearibus obtusiusculis late hyalino marginatis muticis, paleis eis brevioribus breviter bidentatis.

Floret Decembrio, Januario.

Exsicc.: G. Hieronymus: Flora drgentina no. 206 (Koel. Bergi Hieron.). 
Habitat in Argentina: Rio negro cerca Carmen do Patagones (leg. 8. XII. 1874 C. Berg, H. Berol., var. typica m.).

b) var. minor Domin, var. nov.

Subdense caespitosa, culmis gracilioribus humilioribus cum paniculis tantum c. $25-40 \mathrm{~cm}$ altis. sub paniculis brevi tractu minute puberulis, foliis radicalibus numerosis culmos dimidios aequantibus vel superantibus angustioribus ( $1-2 \mathrm{~mm}$ latis) rigidioribus glaucescentibus saepe scabris planis vel complicatis glabrescentibus vel pubescenti-hirsutis, vaginis superioribus saepe glabris, folio culmeo supremo paniculam pro more fulcrante, glumellis subacuminatis.

Argentina: Al pié de los Gigantes; Sierra Achala; Prov. de Córdoba. Leg. 5. XII. 1878 G. Hieronymus (H. Berol.).

c) var. aristulata Domin, var. nov.

Praecedenti similis, sed glumellis e dorso sub apice aristulatis, aristulis c. $0,5-0,75 \mathrm{~mm}$ longis apicem glumellarum pro more haud superantibus.

Argentina: Quebrada al Sur de los Gigantes; Sierra Achala de Córdoba. Leg. 5. XII. 1878 G. Hieronymus (H. Berol.). Ibidem provenit forma aristulis apicem glumellarum paulum superantibus instructis.

subvar. micațula Domin, subvar. nov.

Panicula laxiori plus lobata sed haud interrupta intense nitenti.

Argentina: Quebrada del Rodeo al pié de los Gigantes; Sierra Achala de Córdoba. Leg. G. Hieronymus 28. I. 1881.

d) var. fallacina Domin, var. nov.

Statura robusta cum varietate typica congruens; caespitibus laxis, culmis valde elatis (usque plus $6 \mathrm{dm}$ altis) robustioribus pubescentibus, vaginis infimis patentibus, foliis culmeis laminis elongatis planis usque $5 \mathrm{~mm}$ latis subglabris facie valde scabris instructis, vaginis striatis glabrescentibus (i. e. tantum inter nervos pilis brevibus haud densis obsitis), ligulis sat longis, paniculis robustioribus circa $10 \mathrm{~cm}$ longis conspicue lobatis subdensis nitentibus, spiculis tantum $5 \mathrm{~mm}$ (interdum paulo minus) longis bifloris, glumis glumellisque maxima ex parte scariosis, glumis subaequilongis, superiori acutata flosculos adaequante vel paulum superante, inferiori acuminata flosculis paulo breviori, glumellis muticis.

Argentina: 1. Jucatula cerca de Belen, leg. II. 1872 P. G. Lorentz (H. Berol.).

2. La Ciénaga; Sierra de Tucuman, leg. 10.- 17. I. 1874 Hieronymus et Lorentz.

Habitu Koel. Bergii similis sed notis pluribus gravioris pretii (culmis pubescentibus, spiculis conspicue minoribus) aberrans. Varietates praecedentes multo propius typo accedunt.

4. Koeleria Niederleinii Domin, nov. spec.

Dense caespitosa multiculmis, culmis mediocriter altis vel humiliori bus $($ c. $20-35 \mathrm{~cm})$ totis pubescentibus gracilibus, foliis radicalibus cul- 
meisque inferioribus numerosis mollibus glaucescentibus planis circiter $2-2,75 \mathrm{~mm}$ latis unacum vaginis molliter brevissime pubescenti-velutinis saepius supra glabrescentibus et subtus canescenti-pubescentibus, foliis culmeis more foliorum radicalium planis et eodem modo vestitis, folio culmer supremo paniculam pro more hatud attingente, ligulis circa $1 \mathrm{~mm}$ longis, paniculis cylindricis vel mlongo-eylindricis c. $6 \mathrm{~cm}$ longis angustatis parum lubatis inferne \pm interruptis caleterum subdensis parum nitentihus, spiculis conspieue sed breviter vel brevissime pedunculatis parvis c. $4 \mathrm{~mm}$ vel paulo plus longis bifloris, glumis inaequalibus glaberrimis late hyalinomarginatis superiori latiori acuta flosculis paulo breviori, inferiori angusta acuminati flosculis cirea tertia parte brevirri. glumellis acutatis angustis circa 0.5 vel $0,75 \mathrm{~mm}$ sub apice aristis tenuibus rectis apicem glumellarum nunc attingentibus nunc hatud adaequantibus sed numquam superantibus munitis, paleis breviter bidentatis glumellis subbrevioribus.

Floret Januarin.

Exsicc.: G. Hierunymus et G. Niederlein, Flora Argentina no. 395.

Habitat in Argentinil: Cueva de Perez; Sierra Famatina, Prov. de la Rirjil. Leg. G. Hieronymus et G. Niederlein 26.-28. I. 1879 (H. Berol.).

var. mutica Domin, var. nov.

Differt foliis glabrescentibus angustioribus, glumellis muticis.

Exsicc.: G. Hiernnymus et G. Niederlein, Flora Argentina no. 430.

Habitat in Argentina: En las cercanias de la mina El Cro; Sierra Famatinal: Prov. de la Riojil. Leg. Hieronymus et Niederlein 23,-25. I. 1879 (H. Berol.).

var. pseudo-Bergii Domin, var. nov.

Laxius caespitosa, culmis elatis usque plus $6 \mathrm{dm}$ altis tenuiter pu bescentibus, foliis radicalibus sub flore subsiccis, culmeis elongatis planis usque $4 \mathrm{~mm}$ latis glabrescentibus vel (praesertim subtus) pubescentipuberulis, vaginis omnibus hirsuto-pubescentibus, paniculis c. $7-9 \mathrm{~cm}$ longis valde angustis derisioribus vix lobatis continuis tantum inferne interruptis, spiculis cum typo congruentibus.

Exsicc.: F. Schickendantz, Flora Argentina no. 131.

Argentina: Jucatula cerca Belen; Prov. de Catamarca. Leg. an 1879/1880 F. Schickendantz (H. Berol.).

5. Koeleria Grisebachii Domin, Mag. Bot. Lap. IV, 342 (1904), nomen nudum.

Synon.: K. caudata Griseb., Goet. Abh. XXIV, 292 (1879), non Steudel 1855!

Laxius caespitosa circiter $2 \mathrm{dm}$ alta, toliis radicalibus brevioribus pro more planis c. $1-2 \mathrm{~mm}$ latis parum hirsutis vel glabrescentibus mollioribus viridibus, vaginis glabrescentibu; (i. e. minute puberulis), culmis gracilibus totis retrorsum villosulis, folis culmeis paucis (c. $2-3$ ) laminis brevibus vix $2 \mathrm{~cm}$ longis planis culmo adjacentibus instructis, ligulis brevibus, paniculis cylindricis c. $4-5 \mathrm{~cm}$ longis sublobatis densis, 
ramis paniculae villosulis, spiculis parvis bifloris raro subtrifloris c. $4-4.5 \mathrm{~mm}$ longis, glumis glabris subaequalibus spiculis quarta vel quinta parte brevioribus, superiori paulum longiori latiori acutata usque subobtusa, inferiori angustiori acuminata. glumellis acuminatis violaceo maculatis paulum (c. $0.25 \mathrm{~mm}$ ) sub apice aristulam brevem (usque c. $1 \mathrm{~mm}$ longam) apicem glumellarum superantem rectam gerentibus, flosculo secundo brevepedunculato, pedunculo hirsuto, rhachide haud protracta, flosculo tertio raro evoluto et tunc diminuto mutico.

Floret Januario-Martio.

Exsicc.: G. Hieronymus et G. Lorentz, Flora Argentina no. 81: K. caudulata Gris.

Habitat in Argentina: Nevado del Castillo; Prov, de Salta. Leg. Hieronymus et Lorentz 19.-23. III. 1873 (H. Berol.).

b) var. rijoensis Domin, var. nov.

Differt a typo caespitibus majoribus, folis culmeis infimis et radicalibus subconvolutis rigidioribus plurimis unacum vaginis glaberrimis (sed vaginis foliorum culmeorum breviter pubescentibus), ligulis sublongioribus, culmis altioribus $(3-4 \mathrm{dm}$ altis), paniculis longioribus, spiculis saepe majoribus minus intense coloratis raro fere pallidis, aristis superius insertis.

Exsicc.: G. Hieronymus et G. Niederlein, Flora Argentina no. 599.

Argentina: En las cercanias de la quebrada 2 a 4 leguas mas arriba del Vallecito; Sierra Famatina; Prov. de la Rioja. Leg. Hieronymus et Niederlein 21. I. 1879 (H. Berol.).

Varietas haec a typo valde aberrat, sed congruit nota gravissima aristulis dorsalibus apicem glumellarum superantibus.

c) var. catamarcensis Domin, var. nov.

Caespitibus laxis, culmis valde exaltatis usque plus $6 \mathrm{dm}$ altis gracilibus totis tenuiter pubescentibus, foliis latioribus (c. $2-3 \mathrm{~mm}$ ) mollioribus vix scabriusculis glabrescentibus, vaginis breviter pubescentibus, paniculis vix lobatis angustis densioribus plus nitentibus, spiculis cum typo convenientibus.

Exsicc.: P. G. Lorentz, Flora Argentina no. 651 (pro K. cristata).

Argentina: Jucatula cerca Belen; Prov. Catamarca. Leg. Lorentz II. 1872 (H. Berol.).

6. Koeleria gracilis Pers. var. boliviensis Domin, var. nov.

Culmis humilioribus glabris vel sub panicula minutissime puberulis c. $2 \mathrm{dm}$ altis gracilibus, foliis radicalibus fere obscure viridibus vel tantum subglaucescentibus angustis c. $1 \mathrm{~mm}$ vel paulo plus latis rigidiusculis (nervo medio albo cartilagineo prominulo) planis saepius \pm complicatis vel subconvolutis unacum vaginis glaberrimis conspicue scabris, foliis culmeis circa 3, laminis brevibus culmis adjacentibus subconvolutis unacum vaginis glabris instructis, ligulis c. $1 \mathrm{~mm}$ vel paulo plus longis, lamina folii culmei supremi paniculam attingente, paniculis cylindricis vel oblongo-cylindricis c. $3-5 \mathrm{~cm}$ longis haud densis parum lobatis, spiculis 
parvis c. $4-4,5 \mathrm{~mm}$ longis oblongis bifloris, glumis brevibus inaequilongis sed fere aequilatis flosculis brevioribus fere totis hyalinis acutis glabris, glumellis interdum subobtuse acutis insuper violacen-maculatis et hyalino marginatis.

Exsicc.: Museum botanicum Berolinense no. 2940.

Habitat in Bolivia australis: Es-che-mokro, prov. Tupiza. Leg. Fiebrig, 22. Il. 1904 (H. Berol.).

7. Koeleria pseudocristata Dom. var, andicola Domin, var. nov.

Culmis pubescentibus exaltatis, foliis planis latis, vaginis pubescentibus, panicula densiori.

Exsicc.: G. Mandon: Plantae Andium Boliviensium no. 1359 (1858).

Habitat in Bolivia: Andes. Prov. Larejaca - Viciniis Sorata; Cotani monte chiliecat. Regio temper. 3200-3400 m Leg. Mandon VII. 1858 (H. Kew).

\section{Novitates Florae Bavaricae.1)}

Von Julius Schuster (München).

Die hier aufgeführten Neuheiten der bayerischén Flora sind grösstenteils in den Berichten (Ber. B. B. G.) und Mitteilungen (Mitt. B. B. G.) der Bayerischen Botanischen Gesellschaft ${ }^{2}$ ) beschrieben, und zwar - no. 29 ausgenommen - in deutscher Sprache. Die folgende Zusammenstellung enthält ausser den lateinischen Diagnosen auch einige Berichtigungen.

1. Nuphar centricavatum Schuster (Allg. bot. Zeitschr., 1905, p. 145, c. fig.) $=$ N. pumilum (DC.) Spreng. var. stellatifidum Schuster ined.

Differt a $N$. pum. genuino stigmate profunde stellatifido, mais papillosis \pm 12 ; planta robustior ac maior.

Wesslingersee bei München.

Durch die angegebenen Merkmaie unterscheidet sich diese Varietät erheblich von dem typischen $N$. pum. Immerhin kann ich sie nach genauem Studium eines reichen Laterials nur für eine Lokalrasse und keine Art halten. Namentlich findet sich die Vertiefung in der Mitte der Narbenscheibe - entgegen den Angaben in der Literatur - stets bei $N$. pum., wenn auch nicht immer so auffallend wie hier.

2. Lepidium campestre R. Br. f. prostratum Vollmann in Mitt. B. B. G., 1901, p. 195.

Caulibus numerosis, $20-50 \mathrm{~cm}$ longis, prostratis.

An der Bahn zwischen Eisenstein und Ludwigstal im bayer. Wald.

1) Zusammenstellung schon veröffentlichter zerstreuter Diagnosen.

2) Cfr. Repert. spec. nov, I, pp. 20, 31, 143.

Fedde. 